Artigo / Article

\title{
Leucemia Mielóide Crônica em pediatria. Perspectivas atuais
}

\section{Chronic Myeloid Leukemia in pediatrics patients. Current approach}

Maria Lucia M. Lee

\section{Introdução}

A Leucemia Mielóide Crônica (LMC) é uma patologia rara na infância, representando apenas $2 \%$ a $3 \%$ das leucemias em crianças e adolescentes menores de 15 anos. ${ }^{1,2}$ Como nos adultos, é caracterizada pela presença do cromossomo Philadelphia $(\mathrm{Ph})$. O cromossoma $\mathrm{Ph}$ é o resultado de uma translocação balanceada entre os braços longos do cromossoma 9 e 22 (t( $9 ; 22)(\mathrm{q} 34 ; \mathrm{q} 11))$, levando à fusão dos genes $\mathrm{BRC}$ e $\mathrm{ABL}$. Este novo rearranjo leva à formação de uma nova proteína, com atividade tirosino quinase desregulada.

Em mais de 90\% dos casos de LMC pediátricos e de adultos, o transcrito BCR-ABL resulta em uma proteína de fusão de $210 \mathrm{k} \mathrm{Da}$ (p210). ${ }^{3,4,13}$

Epidemiologicamente, mais de $80 \%$ dos casos são diagnosticados após os 4 anos de idade, sendo observado que mais de $60 \%$ destes ocorrerão em crianças maiores de 10 anos. ${ }^{5,6}$ Existem, entretanto, relatos da ocorrência da doença em lactentes menores de 24 meses de idade. ${ }^{7,8}$ Não apresenta predileção por sexo ou raça, não estando associada a síndromes genética-constitucionais ou a agentes infecciosos. ${ }^{5}$ A exposição a radiação ionizante é, até o momento, o único fator de risco conhecido a se relacionar com o desenvolvimento de LMC . ${ }^{3,4}$

Por não diferir biologicamente da doença do adulto, apresenta manifestações clínicas e evolutivas semelhantes. ${ }^{15}$ Um diagnóstico incidental pode ser realizado em até $23 \%$ dos pacientes, o que é semelhante ao relatado em adultos. Astenia e sintomas associados a esplenomegalia costumam ser os achados mais comuns no grupo pediátrico. ${ }^{5,6}$ A principal diferença observada na apresentação da doença foram as contagens leucocitárias mais elevadas no grupo pediátrico (média $240 \times 10^{9} / \mathrm{L}$ ) em relação às descritas em adultos (média $12-174 \times 10^{9} / \mathrm{L}$ ). ${ }^{6}$

Médica hematologista pediátrica do Instituto de Oncologia Pediátrica - GRAACC/Unifesp-São Paulo-SP

Correspondência: Maria Lucia de Martino Lee

Rua Botucatu 743 - Vila Clementino

04023-062 - São Paulo-SP - Brasil

E-mail: mlucialee@ig.com.br 


\section{Fatores Prognósticos}

Em adultos, vários esforços foram realizados na tentativa de estabelecer, já ao diagnóstico, critérios que auxiliassem a predizer o prognóstico individual de cada paciente. $\mathrm{O}$ mais freqüentemente utilizado foi o proposto por Sokal e colaboradores na década de 90. O escore de Sokal é baseado em uma fórmula matemática que tem como parâmetros a idade, tamanho de baço, porcentagem de blastos no sangue periférico e contagem de plaquetas ao diagnóstico. Os pacientes são então estratificados em três categorias distintas de risco (baixo, intermediário e alto) orientando a decisão terapêtica inicial. ${ }^{9-12}$

A utilidade dos escores prognósticos não foi estabelecida em crianças, limitando seu uso no grupo pediátrico. Apenas o número de blastos periféricos e em medula óssea ao diagnóstico parecem ter algum significado. ${ }^{5,15}$

Entretanto, assim como nos adultos, tornou-se claro que a chave do prognóstico e, conseqüentemente, da sobrevida está vinculada ao padrão de resposta citogética obtida.

\section{Terapêutica}

Uma vez que a LMC constitui um evento raro na infância, as estratégias terapêuticas adotadas provêm da experiência obtida dos estudos clínicos realizados em adultos.

Previamente ao desenvolvimento do inibidor da tirosino quinase, melisato de imatinibe, as opções terapêuticas no grupo pediátrico, incluindo o IFN, eram de benefício limitado. O transplante de medula óssea (TMO) era considerado a única forma de terapia plausível nesta faixa etária com curvas de sobrevida variando entre $65 \%$ a $75 \%$. Entretanto, um número variável de fatores foi demonstrado afetar o sucesso deste procedimento. $\mathrm{Na}$ avaliação dos resultados no grupo de adultos, postergar o TMO por mais de um ano do diagnóstico foi associado com redução na sobrevida livre de doença (SLD), o mesmo sendo observado na análise retrospectiva de estudos multiinstitucionais que incluíram pacientes com menos de 18 anos. ${ }^{16,17}$ Além disto, sempre deve ser considerada mortalidade associada ao procedimento (aproximadamente $20 \%$ ) e o risco de recidiva pós-transplante $(17 \%)^{14}$

Discutir quais os riscos e benefícios do TMO, que até o momento ainda é considerada a única modalidade de tratamento curativa para a LMC em crianças, torna-se necessário, uma vez que novas prerrogativas surgiram com o advento dos inibidores do gen de fusão BRC-ABL.

\section{Agentes citostáticos}

Os agentes citostáticos historicamente utilizados no tratamento paliativo da LMC são a hidroxiuréia e o bussulfano, este já estando proscrito no manejo da LMC.
A hidroxiuréia é um antimetabólico que pode ser utilizada ao diagnóstico com o objetivo de reduzir o volume da doença e conseqüentemente os sintomas associados a hiperleucocitose e esplenomegalia. Em nosso meio ainda é bastante utilizado como medida terapêutica inicial para alívio dos sintomas. A dose inicial varia de 30 a $40 \mathrm{mg} / \mathrm{kg} / \mathrm{dia}$ ou $0,5 \mathrm{a} 1 \mathrm{~g} / \mathrm{m}^{2} / \mathrm{dia}$, com ajustes de acordo com a redução da leucometria. Possui poucos efeitos colaterais, sendo todos reversíveis com a suspensão da droga, entre eles podemos citar: citopenias, azoospermia, amenorréia e, mais raramente, infiltrados pulmonares. Deve ser suspenso quando os níveis de leucócitos estiverem ao redor de 20.0000 - 10.000/ $\mathrm{mm}^{3}$. A droga pode levar à resposta hematológica completa em alguns casos, mas a resposta citogenética é incomum ( $0 \%$ a $5 \%$ dos casos), não possuindo impacto nem na prevenção da progressão da doença, nem no prolongamento substancial da sobrevida. ${ }^{4,5,18,20}$

\section{Interferon}

A introdução do IFN no tratamento da LMC no início da década de 80 alterou a história natural da doença, uma vez que foi a primeira droga que demonstrou ser capaz de levar a respostas citogenéticas significativas, apesar do mecanismo de ação pouco esclarecido (efeito imunomodulador/antiproliferativo). ${ }^{21}$ Os pacientes tratados na fase crônica inicial com IFN obtêm índices de respostas citogenéticas maiores (RCgM) entre 20\% a $40 \%$ e de resposta citogenética completa $(\mathrm{RCgC})$ entre $5 \%$ a $30 \%$, com curvas de sobrevida livre de eventos (SLE) em dez anos de $72 \%$ para este grupo com RCgC.18,22 Sua combinação com baixas doses de citarabina (ARA-C) parece aumentar os índices de RCgC para $25 \%$ a $35 \% 23$; entretanto, em outros estudos esta associação não demonstrou aumentos de significado estatístico. ${ }^{24}$

A dose máxima diária recomendada é de 5 milhões unidades $/ \mathrm{m}^{2} /$ dia, por via subcutânea, iniciando-se com doses menores (3 milhões, três vezes por semana) com aumento gradativo.4,25 O uso da medicação costuma estar associado a graus variáveis de toxicidade. Na maioria dos pacientes, sintomas semelhantes a um quadro gripal costumam ocorrer. Depressão, perda de peso, queda de cabelo, quadros autoimunes, como púrpura trombocitopênica, anemia hemolítica, neuropatias e hipotireoidismo também podem ser observados. ${ }^{18,25}$ Mesmo antes da era dos inibidores da tirosino quinase, o uso da medicação era dificultado por vários fatores, que direta ou indiretamente interferiam na qualidade de vida dos pacientes. Além dos efeitos colaterais, que em muitos casos eram determinantes da interrupção da droga, a necessidade de injeções diárias e a falta de definição do tempo de uso da droga comprometiam a aderência ao tratamento.

Em pediatria, apenas dois estudos foram publicados relatando a experiência com o uso de IFN + citarabina, ambos contando com número bastante restrito de pacientes.

No primeiro estudo, 12 pacientes menores de 18 anos, com LMC em fase crônica e sem doador aparentado compa- 
tível, foram randomizados a receber IFN ( 5 milhões U/m²/dia) ou IFN com ARA-C $\left(20 \mathrm{mg} / \mathrm{m}^{2} / \mathrm{dose}\right.$, por dez dias, $\left.1 \mathrm{x} / \mathrm{mês}\right)$. Seis meses após o início da terapia, uma resposta hematológica completa foi obtida em 5/6 dos pacientes do grupo IFN + ARA-C que foram avaliáveis e em 4/6 do grupo do IFN. Resposta citogenética maior (RCM) foi observada em três pacientes do grupo INF + ARA-C após um ano do início da terapia e em dois do grupo IFN. Remissão citogenética completa foi observada no seguimento de 39 e 50 meses, em dois pacientes do grupo IFN+ARA-C que recebiam a combinação há mais de vinte meses.

Os efeitos colaterais mais comumente observados foram os hematológicos, levando à descontinuação transitória do tratamento em ambos grupos. A ocorrência de dor abdominal e náusea em um dos pacientes foi relacionada ao ARA$\mathrm{C}$, todavia o esquema terapêutico foi mantido. Em adultos recebendo IFN/ ARA-C, a toxicidade gastrointestinal, incluindo náuseas, vômitos, diarréia ou mucosite, também foi associada ao uso da citarabina, sendo uma das principais causas de interrupção do tratamento. No grupo IFN + ARA-C, um paciente apresentou bloqueio átrio-ventricular.

Apesar do limitado número de pacientes, o que não permite uma análise estatística, os resultados obtidos foram similares aos descritos em adultos. ${ }^{15}$

O mesmo grupo, em 2000, iniciou um estudo prospectivo, fase II, com o objetivo de avaliar a eficácia e a tolerância do IFN em combinação com ARA-C em baixas doses, em crianças e adolescentes portadores de LMC em primeira fase crônica, sem doador aparentado. Quatorze pacientes de 12 diferentes centros foram recrutados. Resposta hematológica completa foi obtida em $58 \%$ dos pacientes após um tempo médio de tratamento de três meses, com uma média de duração de 11 meses. Aos 12 meses, índices de RCgM de $50 \%$ foram observados, incluindo $14 \%$ com RCgC, com média de duração de seis meses. A probabilidade de sobrevida livre de progressão (ausência de ocorrência de fases acelerada ou blástica) aos 11 e 13 meses foi de $83 \%$ e $71 \%$, respectivamente. Foi observado um alto índice de abandono do protocolo, decorrente da ausência ou perda da resposta hematológica ou citogenética. Interrupções transitórias por toxicidade ocorreram em $42 \%$ dos pacientes.

Este estudo entretanto, foi desenhado quando a combinação INF/ARA-C ainda era considerada a melhor abordagem para um paciente pediátrico sem doador disponível; ressaltando o autor que na era do mesilato de imatinibe torna-se de limitado interesse o uso desta associação como tratamento de primeira linha da $\mathrm{LMC}$ pediátrica. ${ }^{26}$

É coerente salientar que, em nosso meio, o acesso ao IFN é usualmente mais factível, sendo em muitos locais ainda a primeira opção terapêutica para o tratamento da LMC infantil.

\section{Mesilato de Imatinibe}

O mesilato de imatinibe (MI) é um inibidor relativamen- te específico da tirosino quinase BCR-ABL com eficácia demonstrada em todas as fases na LMC..$^{13,14,39}$ sendo mais efetivo em pacientes com diagnóstico recente. ${ }^{28}$ Os altos índices de respostas citogenéticas completas e moleculares observadas com o uso da droga mudaram a história do tratamento da LMC do adulto..$^{14,17,27}$ Isto foi claramente demonstrado em estudo multicêntrico, internacional, fase III (International Randomized Study of Interferon and STI571 IRIS), onde mais de mil pacientes portadores de LMC em fase crônica e sem tratamentos anteriores foram acompanhados e avaliados em relação a SLE, sobrevida livre de progressão (SLP), sobrevida global (SG), índices de RHC, RCgC e de remissão molecular maior (RMM), redução de mais de $3 \operatorname{logs}$ do transcrito. Numa avaliação de cinco anos após o início do tratamento observa-se uma SLE de $83 \%$, com $93 \%$ dos pacientes livres de progressão da doença e SG de $95 \%$. Aos 60 meses, as taxas de $\mathrm{RHC}, \mathrm{RCgM}$ e $\mathrm{RCgC}$ foram respectivamente de $98 \%$, 92\% e $87 \%$. Para os pacientes que obtiveram RMM, $100 \%$ apresentaram SLP. ${ }^{39}$

Seus excelentes resultados, associados à sua boa tolerância oral e a eventos adversos em geral, leves a moderados, causaram grande impacto na qualidade de vida dos pacientes adultos portadores de LMC, levando a droga a ser adotada como tratamento de primeira linha em muitos países. ${ }^{40}$

Entretanto, ainda existem poucos dados referentes ao uso de MI em crianças portadoras de LMC, apesar de não existirem razões para acreditar que estas responderiam de forma distinta dos adultos. Os poucos estudos realizados até agora incluíram pacientes com falha ou intolerância ao IFN, em fases avançadas da doença (fase acelerada ou blástica) ou em recidivas pós-TMO. ${ }^{13,14}$

Baseados, portanto, na significativa atividade antileucêmica do MI nos estudos em adultos com LMC, em 2001, um estudo fase I com MI foi conduzido pelo Children's Oncology Group (COG) em pacientes pediátricos portadores de leucemias $\mathrm{Ph}+$ refratárias ou recidivadas. O estudo tinha como objetivos determinar a dose ideal da medicação para estudos fases II e III posteriores, avaliar a farmacocinética e a toxicidade da droga em crianças, além de fornecer dados preliminares da atividade antileucêmica do MI nas leucemias $\mathrm{Ph}+$ infantis. Doses de 260 a $570 \mathrm{mg} / \mathrm{m}^{2} / \mathrm{d}$ foram toleradas, não sendo identificada uma dose tolerada máxima. Resposta hematológica completa foi observada em $100 \%$ dos pacientes com LMC em fase crônica (FC) e 83\% destes apresentaram $\mathrm{RCgC}$. Foi constatada uma grande variabilidade farmacocinética interpacientes, provavelmente por variações individuais no CYP3A, que é a principal enzima metabolizadora da droga. Variações nos níveis de proteína plasmática (particularmente, albumina) possivelmente relacionadas com o estado nutricional, também podem contribuir para estas variações interpacientes na disponibilidade da droga. Os efeitos colaterais mais observados neste estudo foram predominantemente leves a moderados e incluíram náuseas (4\%), vômi- 
tos $(3,5 \%)$, fadiga $(3,5 \%)$ e elevação das transaminases $(6 \%)$. Anemia moderada, trombocitopenia e neutropenia foram vistas em $1 / 3$ dos pacientes. Outras toxicidades menos freqüentes (1,5\%) incluíram dor abdominal, cefaléia, ,estomatite,edema e ganho de peso, artralgia /mialgia. ${ }^{13,14}$

Apoiada neste primeiro estudo e nos dados obtidos em adultos, em que se tornaram evidentes os benefícios clínicos e aumento na sobrevida proporcionados pela droga, em 2003 a U.S Food and Drug Administration (FDA) anunciou a aprovação da mesma para crianças portadoras de LMC - $\mathrm{Ph}+$ resistentes ao IFN e/ou com recidiva pós-TMO. A aprovação do IM naquele momento foi citada como a primeira aprovação de uma nova droga no tratamento do câncer infantil em mais de uma década. ${ }^{38}$

Baseados nestes resultados preliminares, em 2001, Millot et al, ${ }^{30}$ iniciaram estudo multicêntrico fase II com o objetivo de avaliar crianças portadoras de LMC em fase avançada, refratária ou intolerante ao IFN ou recidivada pós-TMO. Foram avaliadas as taxas de remissão hematológica, citogenética e molecular, além do momento e duração destas respostas, o grau de tolerância e a sobrevida global. A dose utilizada variou de $260-340 / \mathrm{m}^{2}$ baseada nos resultados do estudo COG que indicaram que estas proporcionavam exposições da droga similares às doses de 400-600 mg em adultos. $^{7}$ Foram observadas respostas significantes, com índices de $\mathrm{RHC}$ e $\mathrm{RCgC}$ de $80 \%$ e $60 \%$, respectivamente e SG em 12 meses de $95 \%$. Em adultos em que o MI foi introduzido após falha de tratamento com IFN, são observadas taxas de RHC e $\mathrm{RCgC}$ de $60 \%$ e $41 \%$ respectivamente, com SG de $95 \%$ em 18 meses. Também foi observado que $50 \%$ das crianças que se encontravam em FC atingiram RMM, sendo que, em 27\%, o transcrito foi indetectável.

Em adultos, o grau de resposta molecular prediz a sobrevida livre de progressão da doença, mas isto ainda não foi possível de avaliar no grupo pediátrico. ${ }^{27} \mathrm{O}$ relato de um lactente de 12 meses com $\mathrm{LMC} \mathrm{Ph}+$, que apresentou $\mathrm{RCgC}$ após 12 meses de terapia com o uso de MI, e que a monitorização molecular por RTQ-PCR aos 12 e 18 meses de tratamento evidenciou resposta molecular maior e completa respectivamente, demonstra um dos primeiros casos pediátricos onde foi observada a eficácia da droga em reduzir o transcrito a níveis indetectáveis em uma criança sem tratamentos anteriores. $^{3}$

O padrão de toxicidade observada nos estudos em crianças relatam graus de toxicidade considerados aceitáveis, não sendo necessário a interrupção da droga. As toxicidades mais relevantes são as hematológicas (7\% dos casos), com incidência ao redor de $20 \%$. Entre as não hematológicas são citadas: náuseas (13\%), vômitos (10\%), infecções (17\%) e rush cutâneo (13\%).

Apesar do MI ser bem tolerado e com remissões citogenéticas e moleculares podendo ser obtidas em uma porcentagem significante de crianças e adolescentes, mesmo com doença em fases avançadas, várias questões ainda ne- cessitam ser respondidas em pediatria. A tolerância a longo prazo, assim como avaliação de efeitos colaterais tardios ainda necessitam ser acompanhados. Já existem relatos de efeitos colaterais menos comuns em pacientes adultos acompanhados há mais tempo, como, por exemplo, glaucoma. ${ }^{29} \mathrm{~A}$ descrição de hipofosfatemia com possíveis alterações ósseas e no metabolismo mineral, ${ }^{41}$ apesar das controvérsias despertadas, necessita ser avaliada nos pacientes pediátricos em uso prolongado da droga.

Não existe até o momento consenso no uso do imatinibe em crianças com LMC - FC inicial. Inúmeras dúvidas em relação o quanto a droga seria a opção terapêutica inicial para todas as crianças, mesmo nas que possuam doador compatível disponível, ou se deveria ser utilizada apenas como droga citorredutora de doença residual mínima enquanto é aguardado o TMO, ainda angustiam os onco-hematologistas pediátricos. Esta questão se faz preemente, uma vez que não existem evidências definidas até o momento de que a droga seja curativa. Além de menos de $10 \%$ dos pacientes tornarem-se abl-bcr negativos; a suspensão da droga em alguns pacientes que o eram acarretou posteriormente em recaída molecular. ${ }^{31,32}$

Por outro lado, uma vez que o prognóstico está associado à velocidade e extensão da resposta citogenética/molecular, a identificação precoce dos mecanismos de resistência também se faz obrigatória no grupo pediátrico. $\mathrm{O}$ mecanismo de resistência primária é considerado aquele em que se observa uma falha na obtenção da resposta desejada dentro dos primeiros 18 meses. Desta forma, é considerada resistência hematológica a falha na obtenção de resposta hematológica completa dentro de 3 a 6 meses após o início da terapia ( $2 \%-4 \%$ dos casos). Há consenso que a falha em obter qualquer nível de resposta citogenética em 6 meses ou ausência de resposta citogenética maior ( $\mathrm{RCgM})$ aos 12 meses de tratamento ou de $\mathrm{RCgC}$ aos 18 meses são evidências de falha primária ao imatinibe e outras estratégias terapêuticas devem ser consideradas. Cerca de $15 \%$ a $25 \%$ dos pacientes preenchem estes critérios dentro de 6 a 18 meses após o início da droga. ${ }^{34,40}$ A monitorização deverá, portanto, ser realizada em todos estes momentos, com o estudo citogenético de medula óssea. A resistência secundária está relacionada com o tempo de uso da droga e vários mecanismos de desenvolvimento têm sido descritos. Sua monitorização está baseada na avaliação molecular dos pacientes, além de avaliações citogenéticas periódicas para a identificação de clones mutantes. Podem ocorrer mutações no gene bcr-abl, amplificação do transcrito e mutações no sítio de ligação. Este mecanismo de resistência secundária é alvo de ação de novas drogas, entre elas, os inibidores de tirosino quinase de segunda geração (nilotinibe, dasatinibe,...) ou de estudos de combinações entre diferentes drogas. ${ }^{28}$

Transplante de Medula Óssea

O TMO ainda é considerada a única abordagem tera- 
pêutica curativa para a LMC, principalmente no grupo pediátrico. ${ }^{13,30,35,36}$ Os resultados são dependentes de vários fatores, entre eles: a idade, fase da doença, tempo da realização após o diagnóstico ( $<12$ meses ou $>12$ meses), tipo e sexo do doador, depleção medular de células $\mathrm{T}$ do doador, soropositividade para o CMV e a ocorrência de DECH. ${ }^{36}$ Ainda existe um risco de mortalidade associado ao procedimento de $15 \%$, mesmo nas condições ideais $4,17,34$ e um risco de mortalidade nos dez anos subseqüentes de $3 \%$ por ano (primeiros cinco anos), caindo para cerca de $1 \%$ por ano de cinco até 25 anos após. ${ }^{37}$

Historicamente, está associado a curvas de sobrevida em cinco anos de $75 \%$ na fase crônica inicial, com uma redução para $40 \%$ na fase acelerada e apenas $10 \%$ na crise blástica. ${ }^{7,34,40} \mathrm{Em}$ crianças, embora exista uma menor experiência em LMC, os resultados parecem ser comparáveis aos observados em adultos com relatos de SLE entre $70 \%-86 \%$, para TMO aparentado realizado dentro dos primeiros dois anos. Resultados comparáveis são observados em TMO não aparentados realizados dentro das mesmas condições, com curvas de SLE entre $70 \%-74 \%$ em cinco anos. ${ }^{16}$

Em uma coorte de 314 pacientes pediátricos de relato do EBMT, o TMO com doador aparentado demonstrou curvas de SLE e SG de $59 \%$ e $71 \%$ respectivamente. Aqueles que realizaram TMO com doador não aparentado apresentaram curvas de SLE de $50 \%$ e SG de $57 \%{ }^{36}$

Conseqüentemente, maiores intervalos entre o diagnóstico e o TMO e inclusão de crianças após a primeira fase crônica resultaram em índices de SLE de 42\%-62\%.

Entretanto, todos os dados refentes ao TMO provêm da era pré-imatinibe. Atualmente, a decisão do TMO passa a ser baseada em três principais variáveis: idade do paciente, grau de resposta ao imatinibe e a disponibilidade de doador. ${ }^{37}$ No grupo pediátrico, os dois últimos ítens provavelmente serão alguns dos critérios orientadores das condutas terapêuticas a serem seguidas em relação a indicação de TMO. Existem, no momento, muitas dúvidas em relação a qual a melhor conduta a ser tomada perante uma criança com diagnóstico de LMC. Através da avaliação de um questionário realizado pelo COG, onde 418 médicos afiliados responderam, podemos avaliar o conflito existente em relação ao tema. Houve uma tendência clara por parte dos oncohematologistas clínicos a utilizar o imatinibe como droga inicial no tratamento da LMC, mesmo em paciente com doador aparentado disponível e até adiar o procedimento em paciente com doador não aparentado. Por outro lado, os especialistas com foco em TMO predominantemente, mantiveram a indicação do procedimento como tratamento inicial, mesmo nos não aparentados. ${ }^{35} \mathrm{Na}$ realidade, dados preliminares não indicam uma influência adversa do imatinibe previamente ao TMO, mas ainda existem dúvidas se nas crianças com doador disponível, postergar o procedimento para além de um ano não diminuiria o potencial de cura da doença. ${ }^{16}$
A recomendação de que a decisão de adiar TMO em paciente pediátrico com doador compatível seja atrelada a protocolo científico clínico que permita caracterizar o paciente com resposta ideal ao imatinibe (RMM), assim como identificar em qualquer momento aquele considerado de alto de risco de progressão da doença e rapidamente encaminhá-lo ao TMO, deve ser uma premissa para o onco-hematologista pediátrico. Assim como os responsáveis pela criança precisam ser informados dos resultados disponíveis com o TMO na era pré-imatinibe e esclarecidos das possibilidades de insucesso com a droga. ${ }^{17}$

$\mathrm{Na}$ avaliação de indicação de TMO em pacientes em uso do MI, devem ser considerados de risco para progressão, aqueles pacientes pediátricos que preencham os critérios de resistência primária ou secundária.

\section{Recomendações}

O onco-hematologista pediátrico não pode mais neste momento ser retrógrado de não reconhecer o impacto indiscutível do MI no tratamento da LMC, mas também não deve ser precipitado e encarar o TMO como uma abordagem terapêutica do passado. Em alguns centros, o TMO já tem sido postergado naqueles pacientes pediátricos que obtêm negativação do transcrito bcr-abl dentro dos primeiros 18 meses do tratamento com o IM. Ficando claro que o mais prudente é a avaliação dinâmica do tratamento , redirecionando a conduta de acordo com a monitorização laboratorial.

Infelizmente, em nosso meio, o tratamento da LMC pediátrica ainda é bastante heterogêneo, uma vez que a disponibilidade de acesso aos recursos não é homogênea entre as regiões de nosso país. Além das dificuldades que ainda existem, seja para obtenção do IFN ou ingresso nos centros especializados em TMO pediátricos, o imatinibe ainda permanece sem liberação na rede pública para indivíduos menores de 18 anos. Desta forma, a grande maioria de nossas crianças com LMC, ainda não podem ser beneficiadas com uma terapia altamente efetiva em impedir a evolução da doença e que abriu as portas para uma nova fase no tratamento do câncer, o tratamento molecular.

\section{Abstract}

Chronic myeloid leukemia (CML) is a rare event in childhood, comprising of less than 5\% of all leukemia cases in this age group. CML is characterized by the presence of a specific molecular marker, the Ph+ chromosome, which is responsible for almost all etiopathogenesis, hence, it has clinical and course characteristics that do not differ from the adult population. In pediatrics the therapeutic approach is based mainly on the experience obtained with adult protocols. With bone marrow transplantation (BMT) being the only cure option, this procedure is more effective in patients with compatible related donors and performed during the initial chronic phase of the disease. The great anti-leukemic efficacy seen with 
imatinib mesylate was responsible for the approval of this drug in pediatric use for intolerant or refractory -interferon treated patients or relapsed patients after BMT. Currently, its use in pediatric patients with recently diagnosed $C M L$, who have a compatible donor, has become a great dilemma. There is no agreement yet on which is the best way to use this drug or even whether it will ever replace BMT. Further studies with longer follow-up periods are still needed. Rev. bras. hematol. hemoter. 2008; 30(Supl.1):59-65.

Key words: Chronic Myeloid Leukemia; pediatric; Imatinib mesylate.

\section{Referências Bibliográficas}

1. Ries LAG, Smith MA, Gurney JG, et al. Cancer incidence and survival among children and adolescents: United States SEER Program 1975-1995. SEER Pediatr Monogr. 1999;99:46-9

2. Stenber CP. Chronic myeloproliferative disorders. In: Mcmillan JA, Oski F. Oski s Pediatrics. 4th ed. Philadelphia, Lippincott Willians \& Wilkins, 2006.

3. Aricò M, Biondi A. Myelodisplastic syndromes and chronic myeloproliferative disorders. In: Pui $\mathrm{CH}$ Childhood Leukemias. Cambridge University Press, 1999.

4. Pasquini R. Leucemia Mielóide Crônica. Variantes de Leucemia Mielóide Crônica. In: Zago MA, Falcão RP, Pasquini R. Hematologia - Fundamentos e Prática. Editora Atheneu, 2001.

5. Altman AJ, Fu C. Chronic leukemias of childhood. In: Pizzo PA \& Poplack DG. Principles \& Practice of Pediatric Oncology. 5th Philadelphia, Lippincott Willians \& Wilkins, 2006

6. Millot F, Traore P, Guilhot T et al. Clinical and biological features at diagnosis in 40 children with chronic myeloid leukemia. Pediatrics. 2005;116:140-43.

7. Richardson MW, Grewal SS. Use of imatinibe mesylate in a 15 month old with Philadelphia chromosome positive $(\mathrm{Ph}+)$ chronic myelogenous leukemia. Pediatr Blood Cancer. 2008;50:186.

8. Arancibia AM, Bendit I, Epelman S. Complete Response to imatinib mesylate treatment in a 12 month - old patient with chronic myeloid leukemia. Pediatric Blood Cancer. 2007 (publicação on line Wiley-Liss,Inc. DOI 10.1002/pbc.21439).

9. Sokal JE, Cox EB, Baccarani M et al. Prognostic discrimination in "good - risk" chronic granulocytic leukemia. Blood 1984; 63: 789-99.

10. Mughal TI, Goldman DM. Chronic myeloid leukemia: STI 571 magnifies the therapeutic dilemma. Eur J Cancer 2001;37:561-68.

11. The Italian Cooperative Study Group of Chronic Myeloid Leukemia. Prognostic factors in chronic myeloid leukemia. Relationship with Interferon and bone marrow transplantation. Leuk Lymphoma. 1993;11:67-91

12. Hasford J, Firmann P Hehlmann R et al. Prognosis and prognostic factors for patients with chronic myeloid leukemia: nontransplant therapy. Semin Hematol. 2003;40:4-12.

13. Kolb EA, Pan Q, Ladanyi M, Steinherz PG. Imatinib mesylate in Philadelphia chromosome - positive leukemia of childhood. Cancer. 2003;98:2643-50.

14. Champagne MA, Capdeville R, Krailo M et al. Imatinib mesylate (STI 571) for treatment of children with Philadelphic chromosome-positive leukemia: results from a Children's Oncology Group phase 1 study. Blood. 2004;104:2655-60.

15. Millot F, Brice $\mathrm{P}$, Philippe $\mathrm{N}$ et al. a-interferon in combination with citarabine in children with Philadelphia chromosome positive chronic myeloid leukemia. J Pediatr Hematol Oncol. 2002;24:18-22.
16. Bolland CM, Krance RA, Heslop He. Hematopoietic stem cell transplantation in pediatric oncology. In: Pizzo PA \& Poplack DG. Principles and Practice of Pediatric Oncology. 5th ed. Philadelphia, Lippincott William \& Wilkins, 2006.

17. Pulsipher MA. Treatment of CML in pediatric patients: Should imatinib mesylate (STI-571, Gleevec) or allogeneic hematopoietic cell transplant be front-line therapy? Pediatric Blood Cancer. 2004; 43:523-33.

18. The Italian Cooperative Study Group of Chronic Myeloid Leukemia. Interferon alpha-2a as compared with conventional chemotherapy for the treatment of chronic myeloid leukemia. N Engl J Med. 1994;330:820-25.

19. Hehlmann R, Heimpel H, Kolb HJ et al. The German CML study comparison of Busulfan vs Hydroxyurea vs Interferon alpha and establishment of prognostic Score 1. Leuk Lymphoma. 1993; 11:159-68.

20. The Italian Cooperative Study Group of Chronic Myeloid Leukemia. Long-term follow-up of the Italian trial of interferon-a vs conventional chemotherapy in chronic myeloid leukemia. Blood. 1998;92:1541-48.

21. Peschel C, Aman MJ, Rudolf G et al. Regulation of the cytokine network by Interferon. A potentional mechanism of Interferon in chronic myelogenous leukemia. Semin Hematol. 1993; 30: 28-31.

22. Bonifazi F, de Vivo A, Rosti G et al. Chronic myeloid leukemia and interferon - a: a study of complete cytogenetic responders. Blood. 2001;98:3074-81.

23. Guillot F, Chastang $\mathrm{C}$, Michaellet $\mathrm{N}$ et al. Interferon alpha-2b combined with cytarabine versus interferon alone in chronic myelogenous leukemia. N Engl J Med. 1997;337:223-28.

24. Tura S. Citarabine increases karyotypic respons in alpha-IFN treated chronic myeloid leukemia patients: results a national prospective randomized trial. Blood. 1998;92:317a

25. Shtalrid M, Lugassy G, Rosensaft J, Berrebi A. Treatment of chronic myeloid leukemia with interferon alpha (Roferon): Results of the Israeli Study Group on CML. Leuk Lymphoma. 1993; $11: 193-97$

26. Millot F, Guillot J, Nelken B et al. Results of a phase II trial testing Interferon-alpha $2 \mathrm{~b}$ and citarabine in children and adolescents with chronic myelogenous leukemia. Pediatr Blood Cancer. 2006; 47:555-59.

27. Kantarjian HM, Cortes JE, O'Brien $\mathrm{S}$ et al. Imatinib mesylate therapy in newly diagnosed patients with Philadelphia chromosome-positive chronic myelogenous leukemia: high incidence of early complete and major cytogenetics responses. Blood. 2003;101:97-100.

28. Kantarjian HM, Talpaz M, Giles F et al. New insigths into the pathophysiology of chronic myeloid leukemia and imatinib resistance. Ann Intern Med 2006;145: 913-23.

29. Hensley MT, Ford JM. Imatinib treatment: Specific issues related to safety, fertility and pregnancy. Semin Hematol. 2003;40:21-5.

30. Millot F, Guillot J, Nelken B et al. Imatinib mesylate is effective in children with chronic myelogenous leukemia in late chronic and advanced phase and in relapse after stem cell transplantation. Leukemia 2006; 20:187-92

31. Rousselot P, Huguet F, Read D et al. Imatinib mesylate discontinuation in patients with chronic myelogenous leukemia in complete molecular remission for more than two years. Blood. 2007; 109:58-60.

32. Morimoto A, Ogami A, Chiyonobu T et al. Early blastic transformation following complete cytogenetic response chronic myeloid leukemia patient treated with imatinib mesylate. J Pediat Hematol Oncol. 2004; 26:320-22. 
33. Shatt N. Medical management of CML. Hematology. 2007;371375

34. Frazer R, Irvine AE, McMullin MF. Chronic Myeloid Leukaemia in the 21 st century. Ulster Med J. 2007;76:8-17.

35. Thornley I, Perentesis JP, Davies SM et al. Treating children with chronic myeloid leukemia in the Imatinib era: A therapeutic dilemma? Med Pediatr Oncol. 2003;41:115-17.

36. Unal S, Fidan G, Tauil B et al. Allogeneic hematopoietic stem cell transplantation in pediatric chronic myelogenous leukemia cases: Hacettepe experience. Pediatric Transplantation. 2007;11: 645-49.

37. Simon W, Segel GB, Lichtman MA. Early allogeneic stem cell transplantation for chronic myelogenous leukemia in the imatinib era: A preliminary assessment. Blood Cells Mol Dis. 2006;37:116-24.

38. FDA Consumer Magazine. U.S. Food and Drug Administration. 2003 (July-Aug): 8.

39. Druker BJ, Guillot F, Stephen G et al. Five-year follow-up of patients receiving imatinib for chronic myeloid leukemia. N Engl J Med. 2006;355:2408-17.

40. Moen MD, Mckeage K, Plosker GL \& Siddiqui. Imatinib. A review of its use in chronic myeloid leukeamia. Drugs. 2007; 67:299-320.

41. Owen S, Halfield A, Hetuak L. Imatinib and altered bone and mineral metabolism. N Engl J Med. 2006;355:627

O tema apresentado e o convite ao autor constam da pauta elaborada pelos co-editores, Professor Ricardo Pasquini e

Professor Cármino Antonio de Souza.

Avaliação: Co-editores e um revisor externo.

Publicado após revisão e concordância do editor.

Conflito de interesse: não declarado.

Recebido: 22/02/2008

Aceito: 25/02/2008 